\title{
FAME: A FOREST ASSESSMENT AND MONITORING ENVIRONMENT SCENARIO
}

\author{
Wim J. Looyen, Frank O. Zeppenfeldt, Jan Cees Venema and Gert van der Burg \\ National Aerospace Laboratory NLR, P.O. Box 153, 8300 AD Emmeloord, The Netherlands \\ Dirk H. Hoekman, Joost J. van der Sanden, Wageningen Agricultural University \\ Nieuwe Kanaal 11, 6709 PA Wageningen, The Netherlands \\ Paul G. Reichert, Food and Agriculture Organization FAO \\ Via delle Terme di Caracalla, 00100 Rome, Italy \\ Eduard Westinga, International Institute for Aerospace Surveys and Earth Sciences ITC \\ P.O. Box 6, 7500 AA Enschede, The Netherlands
}

\begin{abstract}
Effective management of forest resources requires suitable, up-to-date information on the state, extent and distribution of forest. Remote Sensing data can provide this information. To enable the use of remote sensing data for global fores monitoring, provisions are required to allow operational processing of the data to standardized products. A scenario for the development of a Forest Assessinent and Monitoring Environment is presented. Firstly, a pilot RESPAS is developed, a suitable tool for forest management, to compile information on global forests. Secondly, RFSPAS is upgraded to improve its functionality and to intreduce FMS. Thirdly, RESPAS is extended by introducing remote sensing data from newly launched sensors. By introducing this threestep approach, standardized remote sensing products will become available for use in sustainable forest management giving information on the state, extent and distribution of tropical forests.
\end{abstract}

\section{INTRODUCTION}

Upon initiative of FAO, a feasibility study on global operational forest cover monitoring network for FAO using satellite remote sensing (Burg et al, 1992) has been conducted by NLR, WAU and FAO in the framework of the Tropical Forestry Action Plan (TFAP). Within this study a Forest Assessinent and Monitoring Environment (FAME) scenario has been developed, describing a phased development and introduction of a decentralized network for global forest monitoring.

\section{REQUIREMENTS AND PREMISES}

The FAME scenario is based on the following premises:

1. the monitoring infrastructure, FAME will among others include two different types of technical components: a. Forestry Management Systems (FMS), operated for instance by national forestry services to support decision makers, but also to support regional or global applications.

b. REmole Sensing Preprocessing and Archiving Systems (RESPAS) with the purpose to regularly provide the FMS with standard remote sensing products;

The infrastructure of the information system to be developed is proposed to be hierarchical. The subnational and national components form the highest level, the global level forms the root. At subnational level the most detailed information will be available. This information will be used to support the decision making process at this level. At regional level generalized information shall be available on larger areas (at a different scale). This information can be exchanged at the same level as well. The root collates information at global level. The information necessary to support the above mentioned functions includes forestry related, geographical, demographical and socio-economical information, made available at national level, and information based on remote sensing. Remote sensing data will in general not be received at national level, but at lower levels (subregional, regional or global). Thus a data stream is present through the hierarchy from lower to higher levels (see also Venema\&Burg, 1991). Also an information stream exists from higher to lower levels. Two different kinds of sub-systems are distinguished, the first one preprocessing and archiving remote sensing data (RESPAS), the second one performing the extraction of forestry related information from remote sensing data and integration of this information in a GIS-like environınent with historical and field data (FMS). Both sub-systems can exist at various levels (i.e. national, (sub-)regional and global), though initially the RESPAS will be slightly decentralized.

2. quick start of some form of operations;

3. gradual inclusion of algorithms, methods and tools which will emerge from current research programmes (this is particular true for radar data) and which will subsequently be operationalized;

4. ongoing training component for the use of techniques and tools adjusted to the needs of the user;

5. the development of FAME is synchronized to the availability of spaceborne remote sensing sensor systems; 6. parallel development and expansion of the organizational infrastructure to support the global monitoring task.

The evolutionary development scenario of FAME is based on the functions identified as being crucial for the viability of a system: 1) operations, 2) methodology and sensor development and 3) system development.

\section{SHORT TERM DEVELOPMENT PLAN}

It is proposed to develop, as a first step, a pilot RESPAS facility. This pilot facility can be used to regularly produce well defined, standard pre-processed remote sensing products for national forestry services. Experience gained with this pilot facility can be used to assess a 'validated product line' which can subsequently be implemented in/copied to a number of (existing) (sub-)regional facilities. This decentralization of pre-processing allows for an expansion of the system to cover more countries in the mid or long term. 
An obvious way to decrease development time and cost to allow a quick start is to relax performance requirements and functionality. This can be achieved by, among others, limiting the geographical coverage of the pilot facility.

Limiting the geographical coverage of the pilot RESPAS to those countries for which data is most urgently needed will significantly contribute to a reduction of the pre-processing load; hence, a reduction of the initial resources required (computer infrastructure, operators, data and development cost, etc.) can be achieved. As a consequence, the use of already existing and operational (computer) infrastructure can be considered. This option is further strengthened because timeliness requirements and data update frequency are relaxed for operations with this pilot facility.

For this first phase, it is essential to select a number of countries in which sufficient forestry and related remote sensing expertise exists:

- to use the output products of the pilot RESPAS;

- to provide the required feedback.

From a technical point of view, remote sensing imagery is (or will be) available for almost all regions of interest to forestry. More experience is gained in operational forestry services using optical data then in using radar data. However, the predominant cloud cover in the humid tropics often prevents to obtain (multidate) satellite imagery using optical sensors. Therefore, in view of the urgent need for information of these regions, a radar component, apart from an optical component, is essential in the pilot RESPAS facility.

RESPAS will act as an interface between data acquired by a variety of spaceborne remote sensing sensor systems on the one hand (NOAA, ERS-1, LANDSAT, SPOT, JERS-1, ...) and a single user conmunity (national forestry services) on the other. The processing inethods available to date allow the generation of uniform 'zero level' products in a semi automated way. Local differences in observational and applicational circumstances, along with a lack of validated, generally applicable post-processing algorithms prevent an automated, uniform information extraction procedure (e.g. classification) based on these zero level products. However, using knowledge on the local situation, experts can in many cases use these zero level products in their operations provided they are regularly made available in a convenient format, e.g. as high quality photographic products and/or as computer files in the form of maps for visual inspection. Digital data products allow (further) computer assisted analysis, including the use of GIS

Apart from the development of a (centralized) pilot RESPAS facility, it is proposed to assess the needs for dedicated Forest management tools to be included, at a later stage into a Forest Management System (FMS). These will be operated by suh-national, national and/or regional forestry services. Apart from general GIS-like characteristics, these systems include a dedicated set of tools for use in forestry with which the RESPAS output can be further analyzed and merged with other data, results can be archived and retrieved and with which modelling for management support can be performed. The definition of FMS can start parallel to the development of the pilot RESP'AS facility. The digital output of the pilot RESPAS can initially be incorporated in standard GIS systems, if available, or be made available as high quality photographic products.

\section{MID TERM DEVELOPMENT PLAN}

The mid term development plan of FAME comprises the following elements:

1. Enhancement of the functionality of RESPAS.

Automated digital image processing algorithms are currently being developed specially for digital radar data of tropical forests (e.g. TREES and SAREX), and results will be available shortly. In view of these ongoing research activities the functionality of the pilot RESPAS facility can be improved gradually in order to deliver more advanced output products at a later stage. This improved functionality can be achieved by means of a sequence of upgrades. These upgrades are preceded by a short phase during which developed and validated remote sensing based methodology is taken from the research environment and prepared for implementation in the operational RESPAS.

2. Geographical expansion to cover more countries.

Geographical expansion is achieved by decentralization of the remote sensing pre-processing by 'copying' the initial RESPAS to higher levels. Apart from financial constraints, the rate by which decentralization takes place will have to be adapted to specific (sub)-regional technical and infrastructural possibilities. The latter will be different for the various regions involved. It can be envisaged that the initial pilot RESPAS will continue to act as a center from which introduction of experimental RESPAS products in countries will be performed, system developments, modifications and upgrades can be prepared, coordinated and controlled. This decentralization should be controlled by a Programme Advisory Commitee (Burg, et al, 1992 and Looyen et al, 1993)

3. Introduction of FMS.

FMS system development will be started. Meanwhile research on FMS functionality shall be continued.

\section{LONG TERM DEVELOPMENT PLAN}

For wide-scale use of remote sensing data for, say, yearly acquisition of imagery of (tropical) forests on national to global scales the primary requirement seems to be for a medium spatial resolution (about 100 to 500 meter as provided by the RADARSAT system). The latter seems to be a good trade-off between cost of acquiring and processing of data and the information content required at these scales. Especially for the humid tropics a cloud penetrating capability (radar) would be an advantage although a high repeat frequency and/or an equatorial orbit could also minimize cloud cover problems.

A system configuration of FAME which accounts for a medium resolution component in the long term is composed of:

-a high resolution component

-a medium resolution component.

The high resolution component comprises currently planned sensors of which the data is input for the decentralized RESPAS network. User experience gained with products generated by the RESPAS network (using for instance medium resolution RADARSAT data) can assist in the definition of a future medium resolution component of the space segment. 
As to the latter: a design as presented in TREIS (Raney\&Specter, 1991; Burg et al, 1991) might be used as a starting point for the definition of this medium resolution component. The TREIS design concept is based on a P-band SAR (considered optimal for forestry applications) of low data rate. The latter allows the use of (existing) NOAA type data reception facilities which can be integrated in the decentralized RESPAS facilities. The latter can then be upgraded to process and disseminate the medium resolution data as well.

This means that the large amounts of medium resolution data, required to regularly achieve a complete coverage of the region of interest, would be readily available to those who actually use it. Dissemination of this medium resolution data to local end-users, on request complemented with high resolution imagery made available (in a consistent format) through the RESPAS facilities, allows for involving local knowledge in the interpretation of this data to achieve optimal results.

\section{CURRENT STATUS}

FAO, in cooperation with NLR, ITC and WAU are translating the recommendations of the feasibility study, especially the short term development plan, into a project programme proposal for submission to donor countries. This project programme proposal comprises a detailed work breakdown carrying the following activities:

-detailed assessment of user requirements for a pilot Forest Assessment and Monitoring Environment (in particular RESPAS and FMS), requiring active participation of selected countries in South-America, South-East Asia and Africa; -installation of an Project Advisory Committee; -generation and evaluation of optical and radar satellite demonstration products for use in forestry, requiring active feedback from the selected countries; -workshops;

-definition, design and development of the RESPAS facility; -definition of the FMS systems;

-training of operators and decision-makers; -preparation of FAME Programme Proposal;

Parallel to this FAO-activity, the Netherlands Remote Sensing Board (BCRS) have approved a project proposal by ITC, NLR, WAU and FAO, to assess the user requirements for the RESPAS facility. The first item in this project is the preparation of a questionnaire to be adressed to officers of national forest departments. The aim of this questionnaire is to assess:

-the recent problems in monitoring, management, planning and maintenance of forest resources;

-the use of remote sensing data at present;

-the sufficiency of the present data to supply the information demand;

the current constraints and limitations for an efficient and cost-effective use of remote sensing data:

-the possible solutions to overcome the constraints at this moment and in future.

The second item of the project proposal is the assessment of user requirements by visiting a number of tropical countries where the problems associated with e.g. deforestation are evident, where accurate and timely forest information is needed and where a remote sensing infrastructure exists. ITC and FAO have visited Thailand, Indonesia, the Phillipines, Colombia and Guinea. The third item is the generation, by NLR and WAU, of optical and radar satellite demonstration products guided by the user requirements, using existing data, hardware and software. These products will become available as hardcopy photographic products. The fourth item is the presentation of the results and demonstration products for an international forum, at the Kotka-II meeting in Finland of May 1993. The recommendation from this meeting was "Recognizing the need for timely and reliable information on forest resources, the initiave to develop and maintain a global network of remote sensing processing and arcgiving systems was supported. These systems would provide optimized remote sensing data to strengthen the capacity of the countries to assess and monitor their own forest resources as well as assist FRA and TFAP activities. The need for a remote sensing satellite dedicated to global forest monitoring should be examined".

\section{CONCLUSIONS}

At present the use of high resolution optical and radar remote sensing data on subnational scale is being assessed in a large number of projects, but this has not yet resulted in a mature uniform methodology. Moreover, a specification of uniform and validated remote sensing end products suited for extensive use is not available yet. This is particularly true for radar remote sensing data, although there is no doubt as to the usefulness of this data, particularly in the humid tropics. Therefore a dedicated effort should be spent towards the development of uniform operational methods for forest monitoring. To this end a strong interaction is necessary between the system developers, the user community and the research community.

In view of the above a "developing while working" strategy is proposed to come to the realization of a Forest Assessment and Monitoring Environment (FAME). The establishment of a pilot RESPAS is therefore emphasized.

\section{REFERENCES}

Burg et al, 1991: Burg, G. van der, J.B. Borger, J.C. Venema, 1991. Possibilities for a Netherlands contribution towards a low cost dedicated infrastructure for global monitoring of forests, NLR TR $91316 \mathrm{~L}$, NLR, Amsterdam.

Burg et al, 1992: Burg, G. van der, J.C. Venema, D.H. Hoekman, J.J. van der Sanden, P.G. Reichert, 1992. Feasibility study on global operational forest cover monitoring network for FAO using satellite remote sensing, BCRS Report NRSP-2-92-05, BCRS, Delft.

Raney\&Specter, 1991: Raney, R.K., C.N. Specter, 1991. Concept for a user affordable, user friendly radar satellite system for tropical forest monitoring, In: Proceedings IGARSS'91 Symposium, Espoo, Finland, pp 733-736.

Looyen et al, 1993: Looyen, W.J., F.O. Zeppenfeldt, G. van der Burg, J.C. Veneına, D.H. Hoekman, J.J. van der Sanden, P.G. Reichert, E. Westinga, 1993. FAME: a Forest Assessment and Monitoring Environınent Scenario, NLR TP 93047, NLR, Amsterdam.

Venema\&Burg, 1991: Venema, J.C., G. van der Burg, 1991. TFAP: Framework for a feasibility study, Issue II, NLR CR 91037 L, NLR, Amsterdam. 